\title{
Error Estimation in Multicanonical Monte Carlo Simulations With Applications to Polarization-Mode-Dispersion Emulators
}

\author{
Aurenice O. Lima, Member, IEEE, Ivan T. Lima, Jr., Member, IEEE, and Curtis R. Menyuk, Fellow, IEEE
}

\begin{abstract}
This paper shows how to estimate errors in multicanonical Monte Carlo (MMC) simulations using a transitionmatrix method. MMC is a biasing Monte Carlo technique that allows one to compute the probability of rare events, such as the outage probability in optical-fiber communication systems. Since MMC is a Monte Carlo technique, it is subject to statistical errors, and it is essential to determine their magnitude. Since MMC is a highly nonlinear iterative method, linearized error-propagation techniques and standard error analyses do not work, and a more sophisticated method is needed. The proposed method is based on bootstrap techniques. This method was applied to efficiently estimate the error in the probability density function (pdf) of the differential group delay (DGD) of polarization-modedispersion (PMD) emulators that has been calculated using MMC. The method was validated by comparison to the results obtained using a large ensemble of MMC simulations.
\end{abstract}

Index Terms-Multicanonical Monte Carlo (MMC) simulations, optical communications, polarization-mode dispersion (PMD), statistical error.

\section{INTRODUCTION}

$\mathbf{P}$ OLARIZATION-MODE dispersion (PMD) is a major source of impairment in optical-fiber communication systems. Since PMD is a random process, Monte Carlo simulations are often used to compute PMD-related probability distribution functions (pdfs) of quantities like the differential group delay (DGD) and the induced penalty. However, the large PMD penalties that are often of interest to system designers cannot be efficiently computed using unbiased Monte Carlo simulations, since they are very rare. Consequently, Monte Carlo simulations with importance sampling [1] and the multicanonical Monte Carlo (MMC) method [2]-[4], as well as other biasing Monte Carlo methods [5]-[10], have recently been applied to compute

Manuscript received March 11, 2005; revised May 25, 2005. This work was supported in part by the Department of Energy and by the National Science Foundation (NSF). The work of I. T. Lima, Jr. was supported by NSF under Grant 01322899 .

A. O. Lima is with the Department of Computer Science and Electrical Engineering, University of Maryland, Baltimore County, Baltimore, MD 21250 USA, and also with the Department of Electrical and Computer Engineering, North Dakota State University, Fargo, ND 58105-5285 USA (e-mail: alima1@umbc.edu).

I. T. Lima, Jr. is with the Department of Electrical and Computer Engineering, North Dakota State University, Fargo, ND 58105-5285 USA.

C. R. Menyuk is with the Department of Computer Science and Electrical Engineering, University of Maryland, Baltimore County, Baltimore, MD 21250 USA.

Digital Object Identifier 10.1109/JLT.2005.857728 these penalties [11]-[13]. A similar issue arises when calculating the minimum bit error ratio in optical communications systems that are dominated by amplified-spontaneous-emission (ASE) noise and when nonlinear signal-noise and noise-noise interactions are taken into account [14]. It also arises in other problems in optical communications [15], [16]. Hence, the use of biasing Monte Carlo methods is becoming increasingly common.

In contrast to importance sampling and most of the other biasing Monte Carlo methods, MMC does not require prior knowledge of which rare events significantly contribute to the large penalty values in the tail of the pdf. MMC is an iterative method, where in each iteration, it produces a biased random walk that automatically searches the state space for the important rare events. This knowledge is accumulated, allowing the distribution to be obtained further out on the tail from one iteration to the successive iteration. MMC has the additional advantage that it does not require one to combine different biased pdfs of the quantity of interest in order to obtain the entire pdf, as in the case with standard importance sampling. Finally, because the basic algorithm makes relatively little use of the physics of the system to which it is being applied, it is very flexible and can be used with a wide variety of systems.

Since Monte Carlo methods are statistical, error estimates are essential to verify the accuracy of the results. In a previous publication [12], we showed how to compute errors when using importance sampling. In this paper, we show how one can efficiently compute error estimates in MMC simulations using a transition-matrix method. In practice, users of Monte Carlo methods often avoid making detailed error estimates. For example, when using an unbiased Monte Carlo simulation to calculate the pdf of a quantity such as the DGD, the number of samples in each bin of the pdfs' histogram is independent. Hence, when the histogram is smooth, one can infer that the error is acceptably low. This procedure is not reliable with MMC simulations because, as we will show, the MMC algorithm induces a high degree of correlation from bin to bin. While it is our view that it is always best to estimate error with any Monte Carlo method, it is particularly important in MMC simulations.

The goal of any scheme for biasing Monte Carlo simulations, including $\mathrm{MMC}$, is to reduce the variance of the quantities of interest. The MMC uses a set of systematic procedures to reduce the variance, which are highly nonlinear as well as iterative and have the effect of inducing a complex web of 
correlations from sample to sample in each iteration and between iterations. These in turn induce the bin-to-bin correlations in the histograms of the pdfs that we mentioned in the previous paragraph. Thus, calculating the error is significantly more difficult than in standard Monte Carlo simulations. Due to the existing correlations, one cannot apply to MMC the standard error analyses that are traditionally used for simulations with uncorrelated samples. For the same reason, one cannot determine the contribution of the variance from each iteration using standard error-propagation methods as in [12]. Thus, the MMC variance cannot be estimated by applying a standard error analysis to a single MMC simulation. One can, in principle, run many independent MMC simulations in order to estimate error using the standard samplevariance formula [17] on the ensemble of MMC simulations. However, estimating the error of the pdf of the quantity of interest by running many independent MMC simulations is computationally costly and, in many cases, not feasible. One can overcome this problem with the transition-matrix method that we propose. The transition-matrix method is an efficient numerical method to estimate statistical errors in the pdfs computed using MMC. In our method, we use the estimated transition-probability matrix to rapidly generate an ensemble of hundreds of pseudo-MMC simulations, which allows one to estimate errors from only one standard-MMC simulation. The transition-probability matrix, which is computed from a single standard-MMC simulation, contains all the probabilities that a transition occurs from any bin of the histogram of the quantity of interest to any other bin after a step (or perturbation) in the MMC random walk. The pseudo-MMC simulations are then made using the computed transition matrix instead of running full simulations. Each pseudo-MMC simulation must be made with the same number of samples per iteration and the same number of iterations as in the original standard-MMC simulation. Once an ensemble of pseudo-MMC simulations has been calculated, one can use standard procedures to estimate the error. Since the transition matrix that is used in the pseudo-MMC simulations has its own statistical error, it might seem strange at first that it can be used as the basis from which to estimate the error in the MMC simulations. However, bootstrap theory assures us that such is the case [18]. Intuitively, the variation of any statistical quantity among the members of an ensemble of pseudo-MMC simulations is expected to be the same as the variation among members of an ensemble of standard-MMC simulations, because the simulations are of the same type and size.

To illustrate our proposed transition-matrix method, we calculate the pdf of DGD due to PMD and the associated confidence interval for two types of PMD emulators [19]. We validate our method by comparison to the results obtained by using a large ensemble of standard-MMC simulations. We test our method by applying it to PMD emulators, because it was the first random phenomenon in optical-fiber communication to which MMC was applied [3], [4] and has become the sine qua non for testing biasing Monte Carlo methods. Moreover, it is computationally feasible to validate our method with a large ensemble of standard-MMC simulations. That is not the case for most other problems, e.g., the error rate due to optical noise [20] and the residual penalty in PMD-compensated systems [11].

In Section II, we describe the MMC method, its application to PMD emulator studies, and we present results showing the correlations among the histogram bins of the pdf of the DGD. In Section III, we describe the transition-matrix method in detail. Finally, in Section IV, we apply this method to efficiently estimate the error in the pdf of the DGD when using 15- and 80 -section PMD emulators, and we validate it by comparison to a large ensemble of standard-MMC simulations.

\section{Multicanonical Method, PMD Emulators, AND CORRELATIONS}

We briefly review the MMC method proposed by Berg and Neuhaus [2] and show how we implement MMC to compute the pdf of the DGD for two types of PMD emulators. We then present results showing the correlation among the histogram bins of the pdf of the DGD that is generated using the MMC method.

\section{A. MMC Review}

The MMC method is an iterative method, where in each iteration, it produces a biased random walk that automatically searches the state space for the important rare events. Within each iteration, the Metropolis algorithm [21] is used to select samples for the random walk based on an estimated pdf of the quantity of interest or control parameter, which is updated from iteration to iteration. Each new sample in the random walk is obtained after a small random perturbation is applied to the previous sample.

In each MMC iteration, a new histogram of the control parameter is calculated that records how many samples are in each bin. In each iteration, one generates a predetermined number of samples that can vary from iteration to iteration. Typically, each iteration has several thousand samples. Once the predetermined number of samples in any iteration has been generated, the histogram of the control parameter is used to update the estimate of the probability of all the bins as in [2], which will be used to bias the following iteration. After some number of iterations, typically 10-30, the number of samples in each bin of the histogram of the control quantity becomes approximately equal in the range of interest, indicating that the estimated pdf of the control quantity is converging to the true pdf.

\section{B. MMC Implementation to PMD Emulators}

In the computation of the pdf of the DGD, the state space of the system is determined by the random mode coupling between the birefringent sections in an optical fiber with PMD, and the control parameter $E$ is the DGD as in [3] and [4]. When applying MMC, the goal is to obtain an approximately equal number of samples in each bin of the histogram of the control quantity. Here, we compute probabilities by dividing the range of DGD values into discrete bins and constructing a histogram 
of the values generated by the different random configurations of the fiber sections.

The calculations in this paper are based on coarse-step PMD emulators consisting of birefringent fiber sections separated by polarization scramblers [22]. We model the fiber using emulators with $N_{\mathrm{s}}=15$ and $N_{\mathrm{s}}=80$ birefringent sections. Prior to each section, we use a polarization scrambler to uniformly scatter the polarization dispersion vector on the Poincaré sphere. When polarization scramblers are present, the evolution of the polarization dispersion vector is equivalent to a three-dimensional random walk, and an exact solution [23] is available for the pdf of the DGD that can be compared with the simulations. In unbiased Monte Carlo simulations, the unit matrix $\boldsymbol{R}=\boldsymbol{R}_{x}(\phi) \boldsymbol{R}_{y}(\gamma) \boldsymbol{R}_{x}(\psi)$ rotates the polarization dispersion vector before each section, such that the rotation angles around the $x$ axis in the $i$ th section, $\phi_{i}$ and $\psi_{i}$, have their pdfs uniformly distributed between $-\pi$ and $\pi$, while the cosine of the rotation angle $\gamma_{i}$ around the $y$-axis has its pdf uniformly distributed between -1 and 1 . Within each MMC iteration, we use the Metropolis algorithm to make a transition from a state $k$ to a state $l$ by making random perturbations $\Delta \phi_{i}, \Delta \gamma_{i}$, and $\Delta \psi_{i}$ of the angles $\phi_{i}$, $\gamma_{i}$, and $\psi_{i}$ in each section, where $\Delta \phi_{i}, \Delta \gamma_{i}$, and $\Delta \psi_{i}$ are uniformly distributed in the range $[-\varepsilon \pi, \varepsilon \pi]$. To keep the average acceptance ratio close to 0.5 [24], we choose $\varepsilon=0.09$. This perturbation is small, since it does not exceed $10 \%$ of the range of the angles. To account for the correct statistics in $\gamma_{i}$, since the cosine of $\gamma_{i}$ is uniformly distributed in the coarse-step method, we accept the perturbation $\Delta \gamma_{i}$ with probability equal to $\min \left[1, F\left(\gamma_{i}+\Delta \gamma_{i}\right) / F\left(\gamma_{i}\right)\right]$, where $F(\gamma)=$ $0.5\left(1-\cos ^{2} \gamma\right)^{1 / 2}$. When the perturbation is not accepted, we set $\Delta \gamma_{i}=0$. The random variable with acceptance probability given by $\min \left[1, F\left(\gamma_{i}+\Delta \gamma_{i}\right) / F\left(\gamma_{i}\right)\right]$ can be implemented by obtaining a random number from a pdf uniformly distributed between 0 and 1 , and then accepting the perturbation $\Delta \gamma_{i}$ if the random number obtained is smaller than $F\left(\gamma_{i}+\Delta \gamma_{i}\right) / F\left(\gamma_{i}\right)$. To introduce a bias towards large values of the control parameter $E$, each transition from state $k$ to the state $l$ in the iteration $j+1$ is accepted with probability $P_{\text {accept }}(k \rightarrow l)=$ $\min \left[1, P^{j}\left(E_{k}\right) / P^{j}\left(E_{l}\right)\right]$, and rejected otherwise, where $P^{j}(E)$ is the estimate of the pdf of DGD obtained after the first $j$ iterations. At the end of each iteration, we update $P^{j}(E)$ using the same recursion algorithm as in [2], so that the number of hits in each bin of the control-parameter histogram becomes approximately equal as the iteration number increases.

1) Summary of the MMC Algorithm: In the first iteration, we use $M_{1}$ samples and set the pdf of the DGD $P^{1}(E)$ of a PMD emulator with $N_{s}$ sections as uniform, $P^{1}(E)=1 / N_{b}$ ( $N_{b}=$ number of bins). Because every step in the Metropolis algorithm will be accepted with this initial distribution, we more effectively exploit the first iteration by choosing the coefficient of perturbation $\varepsilon=1$. To update the pdf of the DGD at the end of this iteration, we use the recursive equation as in (1), which is the same equation used in any other iteration. We then carry out an additional $N-1$ iterations with $M_{l}(1<l \leq N)$ samples in each iteration. We note that, in general, the number of samples in each iteration does not have to be the same. I now present a pseudocode summary of the algorithm.

Loop over iterations $j=1$ to $N-1$ :

Loop over fiber realizations (samples) $m=1$ to $M_{l}$ :

1) start random walk on $\phi, \gamma$, and $\psi$ with small steps $\Delta \phi, \Delta \gamma$, and $\Delta \psi$

$$
\begin{aligned}
& \Delta \phi=\left\{\Delta \phi_{1}, \ldots, \Delta \phi_{N_{\mathrm{s}}}\right\} \\
& \Delta \gamma=\left\{\Delta \gamma_{1}, \ldots, \Delta \gamma_{N_{\mathrm{s}}}\right\} \\
& \Delta \boldsymbol{\psi}=\left\{\Delta \psi_{1}, \ldots, \Delta \psi_{N_{\mathrm{s}}}\right\}
\end{aligned}
$$

2) compute the provisional value of the DGD $\left(E_{\text {prov }}\right)$ with the angles $\phi+\Delta \phi, \gamma+\Delta \gamma$, and $\psi+\Delta \psi$

3) accept provisional step with probability equal to $\min \left[1, P^{j}\left(E_{m}\right) / P^{j}\left(E_{\text {prov }}\right)\right]$

if step accepted: $E_{m+1}=E_{\text {prov }}$

$$
\begin{aligned}
\phi_{m+1} & =\phi_{m}+\Delta \phi \\
\gamma_{m+1} & =\gamma_{m}+\Delta \gamma \\
\psi_{m+1} & =\boldsymbol{\psi}_{m}+\Delta \boldsymbol{\psi}
\end{aligned}
$$

if step rejected: $E_{m+1}=E_{m}$

$$
\phi_{m+1}=\phi_{m} \quad \gamma_{m+1}=\gamma_{m} ; \quad \psi_{m+1}=\psi_{m}
$$

4) increment the histogram of $E$ with the sample $E_{m+1}$ go to next sample $m$

update the pdf of the DGD $P^{j+1}(E)$

restart histogram

go to next iteration $j$ End

To update $P^{j}(E)$ at the end of each iteration $j$, we use the recursion relations [2]

$$
P_{k+1}^{j+1}=P_{k}^{j+1} \frac{P_{k+1}^{j}}{P_{k}^{j}}\left(\frac{H_{k+1}^{j}}{H_{k}^{j}}\right)^{\hat{g}_{k}^{j}}
$$

where $\hat{g}_{k}^{j}$, the relative statistical significance of the $k$ th bin in the $j$ th iteration, is defined as

$$
\hat{g}_{k}^{j}=\frac{g_{k}^{j}}{\sum_{l=1}^{j} g_{k}^{l}}, \text { with } g_{k}^{j}=\frac{H_{k+1}^{j} H_{k}^{j}}{H_{k+1}^{j}+H_{k}^{j}} .
$$

If $H_{k+1}^{j}+H_{k}^{j}=0$ in a given iteration, then the $k$ th bin has no statistical significance in this iteration. Therefore, we set $g_{k}^{j}=0$ in that iteration. The statistical significance $0 \leq \hat{g}_{k}^{j} \leq 1$ depends on both previous bins and previous iterations, inducing a significant correlation among $P_{k}^{j}$. Finally, the $P_{k}^{j}$ are normalized so that $\sum_{k=1}^{N_{b}} P_{k}^{j}=1$, where $N_{b}$ is the number of bins. MMC is an extension of the Metropolis algorithm [21], where the acceptance rule accepts all the transitions to states with lower probabilities, but rejects part of the more likely transitions to states with higher probabilities. As the number 
of iterations increases, the histogram of the number of hits in each bin will asymptotically converge to a uniform distribution $\left(H_{k+1}^{j} / H_{k}^{j} \rightarrow 1\right)$, and the relative statistical significance will asymptotically converge to zero $\left(\hat{g}_{k}^{j} \rightarrow 0\right)$. Consequently, $P^{j+1}$ will asymptotically converge to the true probability of the control parameter. Equations (1) and (2) were derived by Berg and Neuhaus [2] assuming that the probability distribution is exponentially distributed with a slowly varying exponent that is a function of the control quantity (the temperature in their case and the DGD in this paper). This assumption is valid in a large number of problems in optical-fiber communications, including the pdf of the DGD in fibers with an arbitrary number of sections [3], [4]. The recursions in (1) and (2) were derived by applying a quasi-linear approximation to the logarithm of the pdf, in addition to a method for combining the information in the current histogram with that of previous iterations according to their relative statistical significance [2], [4]. In [3], the author presents a useful code for programming the multicanonical procedure.

\section{Correlations}

As we mentioned earlier, the use of (1) and (2) generates correlated estimates for the $P_{k}^{j}$, although this procedure significantly reduces the variance [2]. In this section, we illustrate this correlation by showing results obtained when we applied MMC to compute the pdf of the DGD for a PMD emulator with 80 sections.

We compute the correlation between bin $i$ and bin $j$ in the histogram of the DGD by doing a statistical analysis on an ensemble of many independent standard-MMC simulations. Suppose that on the $l$ th MMC simulation, we have $P_{i}^{l}$ as the probability of the $i$ th bin and suppose that the average over all $L \mathrm{MMC}$ simulations is $\overline{P_{i}}$. We then define a normalized correlation between bin $i$ and bin $j$ as

$$
C(i, j)=\frac{1}{L-1} \sum_{l=1}^{L} \frac{\left(P_{i}^{l}-\overline{P_{i}}\right)\left(P_{j}^{l}-\overline{P_{j}}\right)}{\sigma_{P_{i}} \sigma_{P_{j}}}
$$

where $\sigma_{P_{i}}$ and $\sigma_{P_{j}}$ are the standard deviations of $P_{i}$ and $P_{j}$, respectively. The normalized correlation defined in (3) is known as Pearson's correlation coefficient [25].

The values for $C(i, j)$ generated by (3) will range from -1 to 1 . A value of +1 indicates a perfect correlation between the random variables. A value of -1 also indicates a perfect anticorrelation between the random variables. A value of zero indicates no correlation between the random variables.

In Figs. 1-3, we show the correlation between bin $i$ and bin $j$ for the DGD in bin $i \mathrm{DGD}_{i}$ equal to 30, 45, and $75 \mathrm{ps,}$ respectively. In this case, we use a PMD emulator with 80 sections and the mean DGD is equal to 30 ps. To compute each value of $C(i, j)$, we used $L=32$ MMC simulations. We compute $\overline{C(i, j)}$ and $\sigma_{C(i, j)}$ using 32 samples of $C(i, j)$. Note that $\mathrm{DGD}_{i}=75 \mathrm{ps}$ represents a case in the tail of the pdf of the DGD, where the unbiased Monte Carlo method has a very low probability of generating samples, by contrast to a biased Monte Carlo method such as MMC. The results show that the correlations are not significant until we use a large value

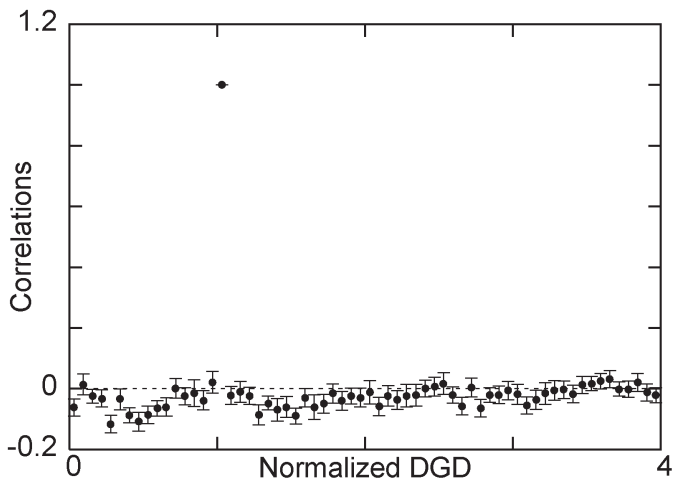

Fig. 1. Correlations between bin $i$ and bin $j$ for the 80-section emulator, where bin $i$ corresponds to $\mathrm{DGD}_{i}=30 \mathrm{ps}(1 \times$ mean DGD $)$. The correlations are computed using 32 standard-MMC simulations. Each standard-MMC simulation consists of $30 \mathrm{MMC}$ iterations with 8000 samples.

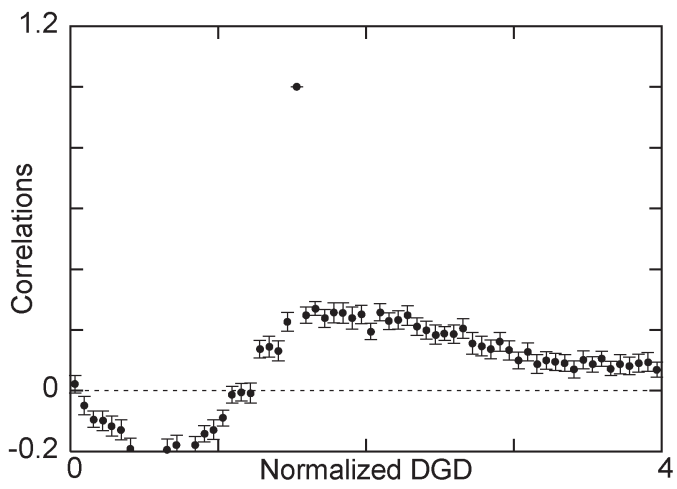

Fig. 2. Correlations between bin $i$ and bin $j$ for the 80-section emulator, where bin $i$ corresponds to $\mathrm{DGD}_{i}=45 \mathrm{ps}(1.5 \times$ mean DGD $)$. The correlations are computed using 32 standard-MMC simulations. Each standard-MMC simulation consists of $30 \mathrm{MMC}$ iterations with 8000 samples.

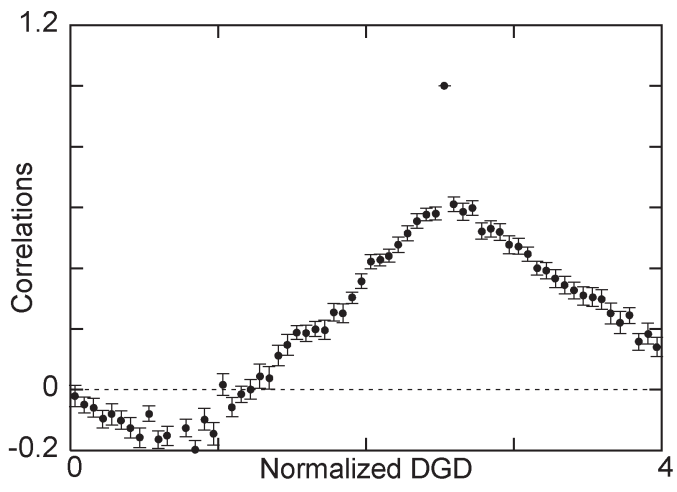

Fig. 3. Correlations between bin $i$ and bin $j$ for the 80 -section emulator, where bin $i$ corresponds to $\mathrm{DGD}_{i}=75 \mathrm{ps}(2.5 \times$ mean DGD $)$. The correlations are computed using 32 standard-MMC simulations. Each standard-MMC simulation consists of $30 \mathrm{MMC}$ iterations with 8000 samples.

for $\mathrm{DGD}_{i}$ compared to the mean DGD, but they do become significant in this limit.

\section{ERror-EstimATION PROCEDURE}

Since MMC uses a procedure to estimate a pdf that is both iterative and highly nonlinear, one cannot apply standard error analyses to MMC. For the same reason, one cannot determine the contribution of the variance from each iteration 
using standard error-propagation methods as in [12]. Here, we introduce an efficient numerical procedure that we refer to as the transition-matrix method to compute statistical errors in MMC simulations that properly accounts for the contributions of all MMC iterations. The procedure is a bootstrapresampling method [18], [26] that uses a computed estimate of the probability of a transition from bin $i$ to bin $j$ of the histogram of the DGD. In a bootstrap method, one estimates a complex statistical quantity by extracting samples from an unknown distribution and computing the quantity. One then repeatedly and independently draws an ensemble of samples from the original sample and computes the statistical quantity of interest using the same procedure by which the statistical quantity was first estimated. One can then estimate the variance of the quantity of interest from a limited sample set using standard techniques. The bootstrap method is applied when it is computationally far more rapid to resample the original sample than to generate new samples, allowing for an efficient estimate of the variance.

\section{A. Bootstrap Method}

Efron's bootstrap [18] is a well-known general-purpose technique for obtaining statistical estimates without making a priori assumptions about the distribution of the data. Suppose one draws a random vector $\mathbf{x}=\left(x_{1}, x_{2}, \ldots, x_{n}\right)$ with $n$ samples from an unknown probability distribution $F$ and one wishes to estimate the error in a parameter of interest $\hat{\theta}=s(\mathbf{x})$. Since there is only one sample of $\hat{\theta}$, one cannot use the sample standard-deviation formula to compute the error. However, one can use the random vector $\mathbf{x}$ to determine an empirical distribution $\hat{F}$ from $F$ (unknown distribution). One can then generate bootstrap samples from $\hat{F}, \mathbf{x}^{\star}=\left(x_{1}^{\star}, x_{2}^{\star}, \ldots, x_{n}^{\star}\right)$, to obtain $\hat{\theta}^{\star}=s\left(\mathbf{x}^{\star}\right)$ by drawing $n$ samples with replacement from $\mathbf{x}$. The quantity $s\left(\mathbf{x}^{\star}\right)$ is the result of applying the same function $s(\cdot)$ to $\mathbf{x}^{\star}$ as applied to $\mathbf{x}$. For example, if $s(\mathbf{x})$ is the median of $\mathbf{x}$, then $s\left(\mathbf{x}^{\star}\right)$ is the median of the bootstrap resampled data set. The star notation indicates that $\mathrm{x}^{\star}$ is not the actual data set $\mathbf{x}$, but rather a resampled version of $\mathbf{x}$ obtained from the estimated distribution $\hat{F}$. Note that one can generate as many bootstrap samples $\mathbf{x}^{\star}$ as one needs, and then generate independent bootstrap-sample estimates of $\hat{\theta}$, $\hat{\theta}_{1}^{\star}=s\left(\mathbf{x}_{1}^{\star}\right), \ldots, \hat{\theta}_{B}^{\star}=s\left(\mathbf{x}_{B}^{\star}\right)$, where $B$ is the total number of bootstrap samples. Then, one can estimate the error in $\hat{\theta}$ using the standard-deviation formula on the bootstrap samples $\hat{\theta}^{\star}$.

The transition-matrix method that we introduce in this paper is related to the bootstrap-resampling method as follows.

1) $\hat{F}$ is an estimate of the transition matrix obtained from a single standard-MMC simulation.

2) $\mathbf{x}_{1}^{\star}, \ldots, \mathbf{x}_{B}^{\star}$ are the collection of samples obtained from the ensemble of pseudo-MMC simulations. We note that $\mathbf{x}_{b}^{\star}$ should be computed using the exact same number of iterations and the exact same number of samples per iteration as in the original standard-MMC simulation.

3) Each $\hat{\theta}_{b}^{\star}$, where $b=1,2, \ldots, B$, is a value for the probability of the $k$ th bin of the histogram of the DGD $p_{k}^{\star}$ obtained from each of the pseudo-MMC simulations.
4) Given that one has $B$ independent $p_{k}^{\star}$, one can obtain an error estimate for each bin in the pdf of the DGD using the traditional sample standard-deviation formula [17], [18]

$$
\sigma_{\hat{\theta}^{\star}}=\left[\frac{1}{B-1} \sum_{b=1}^{B}\left(\hat{\theta}_{b}^{\star}-\overline{\hat{\theta}^{\star}}\right)^{2}\right]^{\frac{1}{2}}
$$

where

$$
\overline{\hat{\theta}^{\star}}=\frac{1}{B} \sum_{b=1}^{B} \hat{\theta}_{b}^{\star} .
$$

\section{B. Transition-Matrix Method}

The transition-matrix method that we introduce in this paper has two parts. In the first part, we obtain an estimate of the pdf of the DGD and an estimate of the one-step transitionprobability matrix $\Pi$. To do so, we run a standard-MMC simulation, as described in Section II-B1. At the same time, we compute an estimate of the transition probability $\pi_{i, j}$, which is the probability that a sample in bin $i$ will move to bin $j$ after a single step in the MMC algorithm. We stress that a transition attempt must be recorded, whether or not it is accepted by the Metropolis algorithm after the fiber undergoes a random perturbation. The transition matrix is a matrix that contains the probability that a transition will take place from one bin to any other bin when applying a random perturbation. It is independent of the procedure for rejecting or accepting samples, which is how the biasing is implemented in the MMC method. An estimate of the transition matrix that is statistically as accurate as the estimate of the pdf using MMC can be obtained by considering all the transitions that were attempted in the MMC ensemble. We use this information to build an $N_{b} \times N_{b}$ one-step transition-probability matrix, where $N_{b}$ is the number of bins in the histogram of the pdf. The transition matrix $\Pi$ consists of elements $\pi_{i, j}$, where the sum of the row elements of $\Pi$ equals 1 . The elements $\pi_{i, j}$ are computed as

$$
\pi_{i, j}=\frac{\sum_{m=1}^{M_{t}-1} I_{i}\left(E_{m}\right) I_{j}\left(E_{m+1}\right)}{\sum_{m=1}^{M_{t}-1} I_{i}\left(E_{m}\right)}, \quad \text { if } \sum_{m=1}^{M_{t}-1} I_{i}\left(E_{m}\right) \neq 0
$$

or $\pi_{i, j}=0$, otherwise. In (6), $M_{t}$ is the total number of samples in the MMC simulation and $E_{m}$ is the DGD sample after $m$ steps. The indicator function $I_{i}(E)$ is chosen to compute the probability of having a DGD sample inside bin $i$ of the histogram. Thus, $I_{i}(E)=1$ inside the DGD range of bin $i$, otherwise $I_{i}(E)=0$.

In the second part of the procedure, we carry out a series of MMC simulations, which we refer to as pseudo-MMC simulations. In each step, if we start, for example, in bin $i$ of the histogram, we pick a new provisional bin $j$ using the probability $\pi_{i, j}$ in the transition matrix $\Pi$. We then accept or reject this provisional transition using the same criteria as in full 
standard-MMC simulations, and the number of samples in the bins of the histogram is updated accordingly. Thus, we are using the transition matrix $\Pi$ to replace the random choice of the $\Delta \phi_{i}, \Delta \gamma_{i}$, and $\Delta \psi_{i}$ that we used in the original standard-MMC simulation. In all other respects, each pseudo-MMC simulation is like the standard-MMC simulation. In particular, the metric for accepting or rejecting a step, the number of samples per iteration, and the number of iterations must be kept the same. It is possible to carry out hundreds of these pseudo-MMC simulations in a fraction of the computer time that it takes to carry out a single standard-MMC simulation. This procedure requires us to hold the entire transition matrix in memory, which could in principle be memory intensive, although this issue did not arise in any of the problems that we considered. This procedure will be useful when evaluating a transition using the transition matrix requires far less computation time. It requires far less computational time than calculating a transition using the underlying physics. That will typically be the case, and was certainly the case, for the problems that we considered.

An estimate of the pdf of the DGD is obtained in the final iteration of each pseudo-MMC simulation. Since the estimates of the probability of each bin in the pseudo-MMC simulations are independent, we may apply the standard formula for the computation of the variance $\sigma_{p_{i}^{\star}}^{2}$ of the $i$ th bin

$\sigma_{p_{i}^{\star}}^{2}=\frac{1}{(B-1)} \sum_{b=1}^{B}\left(p_{i, b}^{\star}-\overline{p_{i}^{\star}}\right)^{2}$, with $\overline{p_{i}^{\star}}=\frac{1}{B} \sum_{b=1}^{B} p_{i, b}^{\star}$

where $p_{i, b}^{\star}$ is the probability of the $i$ th bin in the histogram of the DGD obtained in the $b$ th pseudo-MMC simulation and $B$ is the total number of pseudo-MMC simulations. Thus, $\sigma_{p_{i}^{\star}}$ is an estimate of the error in the $i$ th bin in the histogram of the DGD obtained in a single MMC simulation.

We now illustrate the details of how we choose the provisional transition from bin $i$ to bin $j$ with the following pseudocode:

bin DGD of current sample $=i$

use a random number to generate $x$ from a uniform pdf between 0 and 1: $x \leftarrow U[0,1]$

for $j=1$ to $N_{b}$

if $\left(x<\pi_{i, j}^{\text {cdf }}\right)$

new bin $=j$

break

end if

end for

current bin $=$ new bin

where $\pi_{i, j}^{\mathrm{cdf}}=\sum_{m=1}^{j} \pi_{i, m}$ is the cumulative transition probability.

\section{Assessing the Error in MMC Error Estimation}

The estimate of the MMC variance also has an error, which depends on the number of samples in a single standard-MMC simulation and on the number of pseudo-MMC simulations

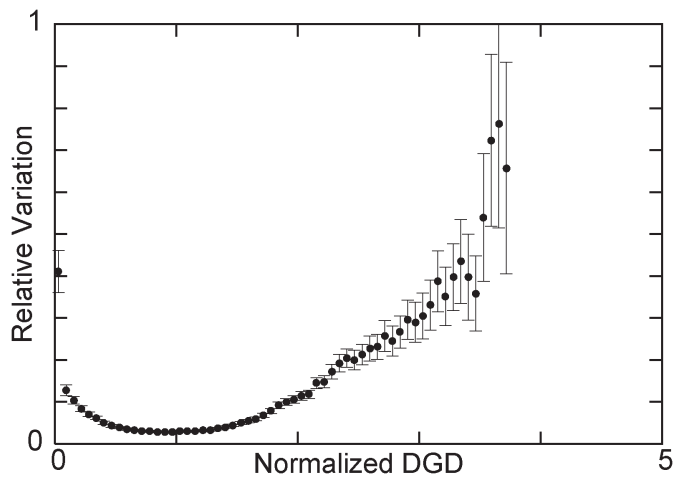

Fig. 4. Relative variation of the pdf of the normalized DGD for the 15-section PMD emulator using 14 MMC iterations with 4000 samples. The symbols show the relative variation $\left(\hat{\sigma}_{\hat{P}_{\mathrm{DGD}}} / \hat{P}_{\mathrm{DGD}}\right)$. The confidence interval is given by (8) when we compute an ensemble of standard deviations using bootstrap resampling for each of the 100 pseudotransition matrices.

(bootstrap samples) [27]. In this paper, the error due to the bootstrap resampling is minimized by using 1000 bootstrap pseudo-MMC simulations. Therefore, the residual error is due to the finite number of samples used to estimate both the pdf of the DGD and the transition matrix in the single standardMMC simulation. Thus, there is a variability in the estimate of the MMC variance due to the variability of the transition matrix $\hat{\boldsymbol{\Pi}}$ as an estimate of the true transition matrix $\boldsymbol{\Pi}$. To estimate the error in the estimate of the MMC variance, we apply a procedure known in literature as bootstrapping the bootstrap or iterated bootstrap [28]. The procedure is based on the principle that if the bootstrap can estimate errors in one statistical parameter using $\hat{\boldsymbol{\Pi}}$, one can also use the bootstrap to check the uncertainty in the error estimate using bootstrapresampled transition matrices $\hat{\Pi}^{\star}$.

The procedure consists of the following.

1) Run one standard-MMC simulation.

2) Generate $N_{B}=100$ pseudo-MMC simulations and computing transition matrices for each of the pseudo-MMC simulations. Therefore, we obtain $N_{B}$ transition matrices that we call pseudotransition matrices $\hat{\Pi}_{B}^{\star}$.

3) For each pseudotransition matrix $\hat{\boldsymbol{\Pi}}_{B}^{\star}$, we calculate $N_{B}=100$ pseudo-MMC simulations $\left(N_{B}\right.$ values for the probability of any given bin of the pdf of the DGD $p^{\star \star}$ ). The double-star notation indicates quantities computed with bootstrap resampling from a pseudotransition matrix. We then estimate the error for the probability of any given bin in the pdf of the DGD $\sigma_{p^{\star \star}}$ for each pseudotransition matrix.

4) Since we have $N_{B}=100$ pseudotransition matrices, we repeat step $3 N_{B}$ times and obtain $N_{B}$ values for $\sigma_{p^{\star \star}}$. Then, we compute the double-bootstrap confidence interval $\Delta p^{\star \star}$ of the relative variation of the error of $p$ (statistical error in $p$, where $p$ is the probability of any given bin in the pdf of the DGD computed using a standard-MMC simulation):

$$
\Delta p^{\star \star}=\left[\frac{\overline{\sigma_{p^{\star \star}}}-\sigma_{\left(\sigma_{p^{\star \star}}\right)}}{p}, \frac{\overline{\sigma_{p^{\star \star}}}+\sigma_{\left(\sigma_{p^{\star \star}}\right)}}{p}\right]
$$




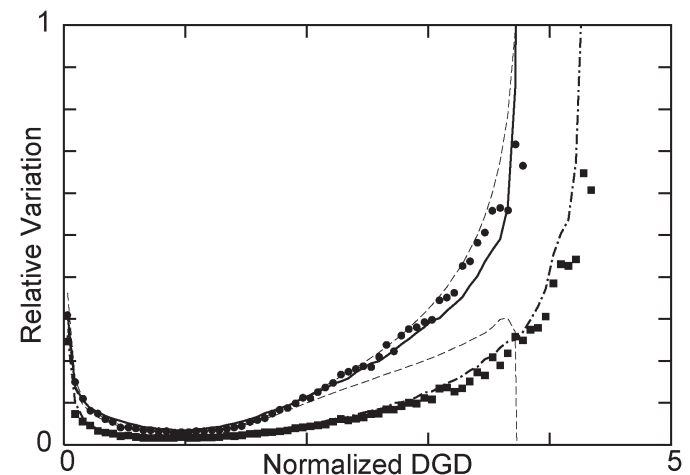

Fig. 5. Relative variation $\left(\hat{\sigma}_{\hat{P}_{\mathrm{DGD}}} / \hat{P}_{\mathrm{DGD}}\right)$ of the pdf of the normalized DGD. 1) Circles: Transition-matrix method based on a single standard-MMC simulation for the 15-section PMD emulator. 2) Solid: $10^{3}$ standard-MMC simulations for the 15-section emulator. 3) Dashed: Confidence interval of the relative variation of the error estimated using the transition-matrix method for the 15-section PMD emulator. 4) Squares: Transition-matrix method based on a single standard-MMC simulation for the 80-section PMD emulator. 5) Dotdashed: $10^{3}$ standard-MMC simulations for the 80-section PMD emulator.

where

$$
\sigma_{\left(\sigma_{p^{\star \star}}\right)}=\left[\frac{1}{N_{B}-1} \sum_{n=1}^{N_{B}}\left(\sigma_{p^{\star \star}}^{(n)}-\overline{\sigma_{p^{\star \star}}}\right)^{2}\right]^{\frac{1}{2}}
$$

and

$$
\overline{\sigma_{p^{\star \star}}}=\frac{1}{N_{B}} \sum_{n=1}^{N_{B}} \sigma_{p^{\star \star}}^{(n)}
$$

In (9) and (10), $\sigma_{p^{\star \star}}^{(n)}$ is the standard deviation of $p^{\star \star}$ computed using the $n$th pseudotransition matrix.

In Fig. 4, we show the relative variation of $p^{\star \star}$ and its confidence interval $\Delta p^{\star \star}$ when we use a PMD emulator with 15 sections and 14 MMC iterations with 4000 samples each (a total of 56000 samples). The confidence interval of the relative variation is defined in (8). As expected, we observe that the error in the estimate of the MMC variance is large when the MMC variance is also large. We conclude that the estimate of the relative variation of the probability of a bin is a good estimate of its own accuracy. This result is similar to what is observed with the standard analysis of standard Monte Carlo simulations [17]. Intuitively, one expects the relative error and the error in the estimated error to be closely related because both are drawn from the same sample space.

\section{APPLICATION AND VALIDATION}

We estimate the pdf of the normalized DGD ( $\left.\hat{P}_{\mathrm{DGD}}\right)$ and its associated confidence interval $\Delta \hat{P}_{\mathrm{DGD}}$ for PMD emulators comprised of 15 and 80 birefringent fiber sections with polarization scramblers at the beginning of each section. The normalized DGD is defined as the DGD divided by its mean value. We use 14 MMC iterations with 4000 samples each to compute the pdf of the normalized DGD when we use a 15-section emulator, and $30 \mathrm{MMC}$ iterations with 8000 samples each when we use an 80-section PMD emulator.

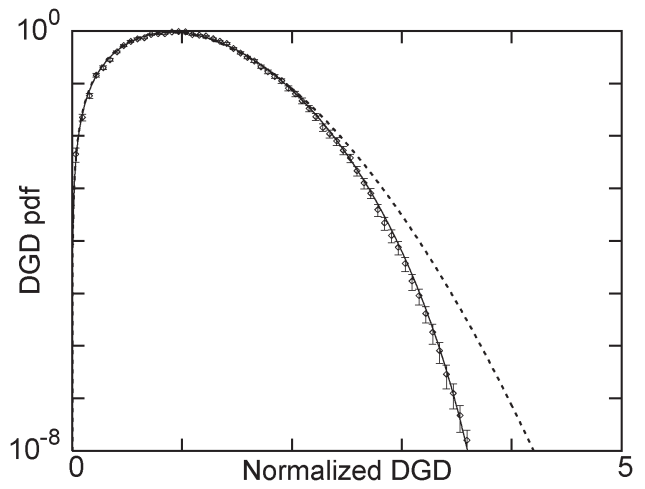

Fig. 6. pdf of the normalized DGD for the 15-section PMD emulator using 14 MMC iterations with 4000 samples. 1) Diamonds: DGD pdf with error estimation using the transition-matrix method. 2) Dashed line: Maxwellian pdf. 3) Solid line: Analytical pdf of the DGD for the 15-section PMD emulator.

We monitor the accuracy of our computation by calculating the relative variation of the pdf of the normalized DGD. The relative variation of the probability of a bin is defined as the ratio between the standard deviation of the probability of the bin and the probability of the bin $\left(\hat{\sigma}_{\hat{P}_{\mathrm{DGD}}} / \hat{P}_{\mathrm{DGD}}\right)$. In Fig. 5, we show the relative variation when we use PMD emulators with 15 and with 80 birefringent sections. The symbols show the relative variation when we apply the procedure that we described in Section III with 1000 pseudo-MMC simulations based on a single standard-MMC simulation and the transition-matrix method, while the solid and the dot-dashed lines show the relative variation when we use 1000 standardMMC simulations. The circles and the solid line show the results for a 15-section PMD emulator, while the squares and dot-dashed line show the results when we use an 80-section PMD emulator. As expected, the result from an ensemble of pseudo-MMC simulations shows a systematic deviation from the result of an ensemble of standard-MMC simulations for both emulators. The systematic deviation changes, depending on which standard-MMC simulation is used to generate the pseudoensemble.

In Fig. 5, the two dashed lines show the confidence interval of the relative variation with the 15-section PMD emulator computed using the transition-matrix method, i.e., the confidence interval for the results that are shown with the circles. While the relative variation that is computed using the transition-matrix method from a single MMC simulation will vary from one standard-MMC simulation to another, the results obtained from different standard-MMC simulations are likely to be inside this confidence interval with a well-defined probability. The confidence interval of the relative variation was obtained using a procedure similar to the one discussed in Section III-C, except that we computed the relative variation of the probability of a bin using the transition-matrix method for every one of the 1000 standard-MMC simulations. Therefore, we effectively computed the true confidence interval of the error estimated using the transition-matrix method. We have verified that the confidence interval calculated using the doublebootstrap procedure on a single standard-MMC simulation agrees well with the true confidence interval in all the cases that we investigated. 


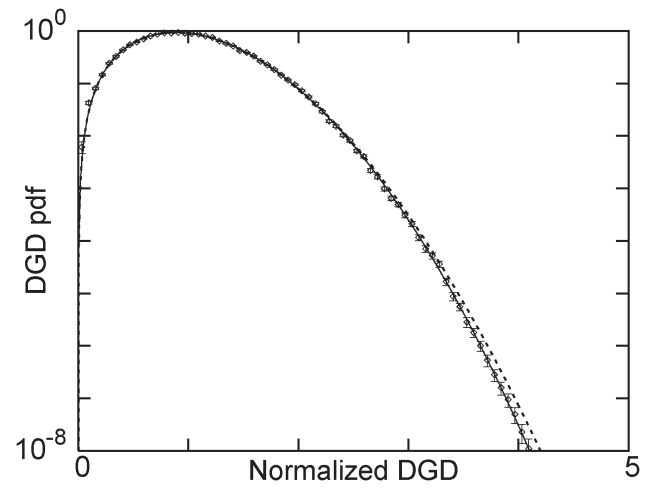

Fig. 7. pdf of the normalized DGD for the 80-section PMD emulator using 30 MMC iterations with 8000 samples. 1) Diamonds: DGD pdf with error estimation using the transition-matrix method. 2) Dashed line: Maxwellian pdf. 3) Solid line: Analytical pdf of the DGD for the 80-section PMD emulator.

We observed excellent agreement between the results obtained with the transition-matrix method based on a single standard-MMC simulation and the results obtained with 1000 standard-MMC simulations for both the 15- and 80-section PMD emulators when the relative error is smaller than $15 \%$. For a larger relative error, the true error is within the confidence interval of the error, which can be estimated using the doublebootstrap method that we described earlier in Section III-C. The curves for the 80-section PMD emulator have a larger DGD range because a fiber with 80 birefringent sections is able to produce larger DGD values than is possible with a fiber of 15 birefringent fiber sections [19].

In Figs. 6 and 7, we show with symbols the results for the pdf of the normalized DGD and its confidence interval using the numerical procedure that we presented in Section III. The solid line shows the pdf of the normalized DGD obtained analytically using the solution presented in [23] for 15 and 80 concatenated birefringent fiber sections with equal length. For comparison, we also show the Maxwellian pdf for the same mean DGD. For both 15- and 80-section emulators, we find that the MMC yields estimates of the pdf of the normalized DGD with a small confidence interval. We used only $56000 \mathrm{MMC}$ samples to compute the pdf of the DGD in a 15-section emulator, but we were able to accurately estimate probabilities as small as $10^{-8}$. Since the relative error in unbiased Monte Carlo simulations is approximately given by $N_{I}^{-1 / 2}$, where $N_{I}$ is the number of hits in a given bin, it would be necessary to use on the order of $10^{9}$ unbiased Monte Carlo samples to obtain a statistical accuracy comparable to the results that we show in the bin with lowest probability in Figs. 6 and 7.

We conclude by stressing that the computational time that is required to estimate the errors using the transition-matrix method does not scale with the time needed to carry out a single standard-MMC simulation. It takes approximately $17.5 \mathrm{~s}$ of computation using a Pentium 4.0 computer with $3 \mathrm{GHz}$ of clock speed to estimate the errors in the pdf of the DGD for the 80-section emulator using 1000 pseudo-MMC simulations with the transition-matrix method, once the transition matrix is available. The computational time that is required to compute the pdf of the DGD using only one standard-MMC simulation requires $60 \mathrm{~s}$ of CPU time. To obtain 1000 standardMMC simulations would require about $16.6 \mathrm{~h}$ of CPU time in this case.

\section{CONCLUSiON}

It is difficult to estimate the statistical errors in multicanonical Monte Carlo (MMC) simulations because the algorithm is iterative and highly nonlinear. We introduce a transition-matrix method that allows us to efficiently estimate the statistical errors from a single standard-MMC simulation, and we show that this method is a variant of the bootstrap procedure. We then applied this method to calculate the probability distribution function (pdf) and its expected error for 15- and 80-section polarizationmode dispersion (PMD) emulators. Finally, we validated this method in both cases by comparing the results to estimates of the error from ensembles of 1000 independent standardMMC simulations. The agreement was excellent. We anticipate that the transition-matrix method will allow one to estimate errors with any application of MMC, including the computation of the pdf of the PMD-induced penalty [11], the pdf of the received voltage in optical communication systems [20], and the computation of rare events in coded communication systems [29].

\section{ACKNOWLEDGMENT}

The authors gratefully acknowledge the helpful participation of Dr. J. Zweck in discussions throughout the course of this investigation.

\section{REFERENCES}

[1] S. L. Fogal, G. Biondini, and W. L. Kath, "Multiple importance sampling for first- and second-order polarization-mode dispersion," IEEE Photon. Technol. Lett., vol. 14, no. 9, pp. 1273-1275, Sep. 2002.

[2] B. A. Berg and T. Neuhaus, "The multicanonical ensemble: A new approach to simulate first-order phase transitions," Phys. Rev. Lett., vol. 68, no. 1, pp. 9-12, 1992.

[3] D. Yevick, "Multicanonical communication system modelingApplication to PMD statistics," IEEE Photon. Technol. Lett., vol. 14, no. 11, pp. 1512-1514, Nov. 2002.

[4] - "The accuracy of multicanonical system models," IEEE Photon. Technol. Lett., vol. 15, no. 2, pp. 224-226, Feb. 2003.

[5] W. K. Hastings, "Monte Carlo sampling methods using Markov chains and their applications," Biometrika, vol. 57, no. 1, pp. 97-109, 1970.

[6] F. Wang and D. P. Landau, "Efficient, multiple-range random-walk algorithm to calculate the density of states," Phys. Rev. Lett., vol. 86, no. 10, pp. 2050-2053, 2001.

[7] D. Yevick and W. Bardyszewski, "A random walk procedure for evaluating probability distribution functions in communication systems," IEEE Photon. Technol. Lett., vol. 16, no. 1, pp. 108-110, Jan. 2004.

[8] J. Wang, "Flat histogram Monte Carlo method," Physica, A, vol. 281, no. 1 , pp. $147-150,2000$

[9] W. Janke and T. Sauer, "Multicanonical multigrid Monte Carlo method," Phys. Rev. E, Stat. Phys. Plasmas Fluids Relat. Interdiscip. Top., vol. 49, no. 4, pp. 3475-3479, 1994.

[10] J. S. Liu and R. Chen, "Sequential Monte Carlo methods for dynamic systems," J. Amer. Stat. Assoc., vol. 93, no. 443, pp. 1032-1044, 1998.

[11] A. O. Lima, I. T. Lima, Jr., J. Zweck, and C. R. Menyuk, "Efficient computation of PMD-induced penalties using multicanonical Monte Carlo simulations," in Proc. Eur. Conf. Optical Communication (ECOC), Rimini, Italy, 2003, pp. 538-539.

[12] A. O. Lima, I. T. Lima, Jr., C. R. Menyuk, G. Biondini, B. S. Marks, and W. L. Kath, "Statistical analysis of the performance of PMD compensators using multiple importance sampling," IEEE Photon. Technol. Lett., vol. 15 , no. 12 , pp. 1716-1718, Dec. 2003. 
[13] M. Secondini, E. Forestieri, and G. Parti, "Markov Chain Monte Carlo technique for outage probability evaluation in PMD-compensated systems," in Proc. Tyrrhenian Int. Workshop Digital Communications, Pisa, Italy, 2004, pp. 1-8.

[14] C. D. Poole and J. Nagel, Optical Fiber Telecommunications, vol. III-A. San Diego, CA: Academic, 1997.

[15] I. Neokosmidis, T. Kamalakis, A. Chipouras, and T. Sphicopoulos "Estimation of the four-wave mixing noise probability-density function by the multicanonical Monte Carlo method," Opt. Lett., vol. 30, no. 1, pp. 11-13, 2005.

[16] T. Kamalakis, D. Varoutas, and T. Sphicopoulos, "Statistical study of in-band crosstalk noise using the multicanonical Monte Carlo method," IEEE Photon. Technol. Lett., vol. 16, no. 10, pp. 2242-2244, Oct. 2004.

[17] R. Walpole and R. Myers, Probability and Statistics for Engineers and Scientists. New York: Macmillan, 1993

[18] B. Efron, "Bootstrap methods: Another look at the Jackknife," Ann. Stat., vol. 7, no. 1, pp. 1-26, 1979.

[19] I. T. Lima, Jr., R. Khosravani, P. Ebrahimi, E. Ibragimov, A. E. Willner, and C. R. Menyuk, "Comparison of polarization mode dispersion emulators," J. Lightw. Technol., vol. 19, no. 12, pp. 1872-1881, Dec. 2001.

[20] R. Holzlöhner and C. R. Menyuk, "The use of multicanonical Monte Carlo simulations to obtain accurate bit error rates in optical communications systems," Opt. Lett., vol. 28, no. 20, pp. 1894-1897, 2003.

[21] N. Metropolis, A. W. Rosenbluth, M. N. Rosenbluth, A. H. Teller, and E. Teller, "Equation of state calculations by fast computing machines," J. Chem. Phys., vol. 21, no. 6, pp. 1087-1092, 1953.

[22] D. Marcuse, C. R. Menyuk, and P. K. A. Wai, "Application of the Manakov-PMD equation to studies of signal propagation in optical fibers with randomly varying birefringence," J. Lightw. Technol., vol. 15, no. 9, pp. 1735-1746, Sep. 1997.

[23] M. Karlsson, "Probability density functions of the differential group delay in optical fiber communication systems," J. Lightw. Technol., vol. 19, no. 3, pp. 324-331, Mar. 2001.

[24] M. H. Kalos and P. A. Whitlock, Monte Carlo Methods. New York: Wiley, 1986.

[25] S. Kachigan, Multivariate Statistical Analysis: A Conceptual Introduction. New York: Radius, 1991

[26] K. Singh, "On the asymptotic accuracy of Efron's Bootstrap," Ann. Stat., vol. 9, no. 6, pp. 1187-1195, 1981.

[27] B. Efron and R. Tibshirani, An Introduction to the Bootstrap. New York: Chapman and Hall, 1993.

[28] J. Booth and P. Hall, "Monte Carlo approximation and the iterated bootstrap," Biometrika, vol. 81, no. 2, pp. 331-340, 1994.

[29] R. Holzlöhner, A. Mahadevan, C. R. Menyuk, J. M. Morris, and J. Zweck, "Evaluation of the very low BER of FEC codes using dual adaptive importance sampling," Comm. Lett., vol. 9, no. 2, pp. 163-165, 2005.

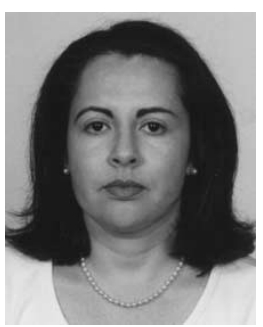

Aurenice O. Lima (SM'00-M'05) received the B.Sc. degree in electrical engineering from the Federal University of Bahia (UFBA), Salvador, Brazil, in 1995, the M.Sc. degree in electrical engineering from the State University of Campinas (UNICAMP), Campinas, Brazil, in 1998, and the Ph.D. degree in electrical engineering from the University of Maryland, Baltimore County, in 2005.

From 2000 to 2005, she was a Research Assistant with the Optical Fiber Photonics Laboratory at the University of Maryland, Baltimore County. In 2005, she became an adjunct faculty member with the Department of Electrical and Computer Engineering, North Dakota State University, Fargo, and with the Mathematics Deparment, Minnesota State University, Moorhead. Her current research interests include modeling and statistical analysis of polarization effects and signal processing for optical fiber communication systems. She is currently focusing her research on the study of advanced Monte Carlo methods for computation of penalties induced by polarization mode dispersion in optical fiber transmission systems. She has authored or co-authored 12 archival journal publications and 21 conference contributions.

Dr. Lima received the Venice Summer School on Polarization Mode Dispersion Award in 2003. In 1996 and 1998, she received Graduate Scholarships from the Brazilian Ministry of Education and from the Brazilian Ministry of Science and Technology, respectively. She is a member of the IEEE Lasers and Electro-Optics Society (LEOS) and of the IEEE Women in Engineering Society.

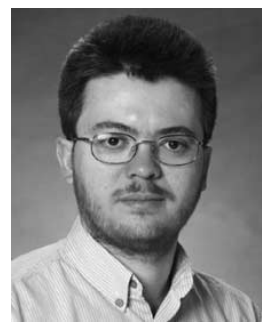

Ivan T. Lima, Jr. (S'95-M'04) received the B.Sc. degree in electrical engineering from the Federal University of Bahia (UFBA), Salvador, Brazil, in 1995, the M.Sc. degree in electrical engineering from the State University of Campinas (UNICAMP), Campinas, Brazil, in 1998, and the Ph.D. degree in electrical engineering in the field of photonics from the University of Maryland, Baltimore County, in 2003 .

From 1986 to 1996, he was with Banco do Brasil (Bank of Brazil), where he served as the Information Technology Adviser of the State Superintendence of Bahia, Brazil. From 1998 to 2003, he was a Research Assistant with the Optical Fiber Photonics Laboratory, University of Maryland, Baltimore County. In 2003, he became an Assistant Professor with the Department of Electrical and Computer Engineering, North Dakota State University (NDSU), Fargo. His research interests have been devoted to the modeling of polarization effects and receivers in optical fiber communications systems and the development of lasers for applications in biotechnology. He has authored or coauthored 22 archival journal papers, 38 conference contributions, one book chapter, and one U.S. Patent.

Dr. Lima received IEEE LEOS Graduate Student Fellowship Award, and he was co-recipient of the Venice Summer School on Polarization Mode Dispersion Award. In February 2004, he was co-instructor of the Short Course (SC210: Hands-on polarization measurement workshop), which was offered at the Optical Fiber Communications Conference and Exposition (OFC) 2004 Los Angeles, CA. In March 2005, he also served as co-instructor of the short courses (SC210-A and SC210-B) at OFCNFOEC 2005, Anaheim, CA. These short courses were sponsored by the IEEE Lasers and Electro-Optics Society (LEOS) and by the Optical Society of America (OSA). He is a member of the IEEE-LEOS and of the OSA.

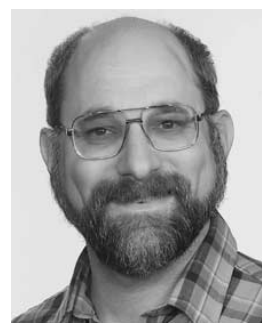

Curtis R. Menyuk (SM'98-F'98) was born on March 26, 1954. He received the B.S. and M.S. degrees from the Massachusetts Institute of Technology, Cambridge, in 1976 and the Ph.D. degree from the University of California at Los Angeles in 1981.

$\mathrm{He}$ has worked as a research associate at the University of Maryland, College Park and at Science Applications International Corporation, McLean, VA. In 1986, he became an Associate Professor with the Department of Electrical Engineering, University of Maryland, Baltimore County (UMBC), where he was the founding member of this department. In 1993, he was promoted to Professor. He was on partial leave from UMBC from Fall 1996 until Fall 2002. From 1996 to 2001, he worked part-time for the Department of Defense, co-directing the Optical Networking program at the DoD Laboratory for Telecommunications Sciences, Adelphi, MD, from 1999 to 2001. From 2001 to 2002, he was Chief Scientist at PhotonEx Corporation. For the last 18 years, his primary research area has been theoretical and computational studies of lasers, nonlinear optics, and fiber optic communications. He has authored or coauthored more than 190 archival journal publications as well as numerous other publications and presentations. He has also edited three books. The equations and algorithms that he and his research group at UMBC have developed to model optical fiber systems are used extensively in the telecommunications and photonics industry.

Dr. Menyuk is a member of the Society for Industrial and Applied Mathematics and the American Physical Society. He is a Fellow of the Optical Society of America. He is a former UMBC Presidential Research Professor. 